\title{
Converting PSO dynamics into complex network - Initial study
}

Michal Pluhacek, Jakub Janostik, Roman Senkerik, and Ivan Zelinka

Citation: AIP Conference Proceedings 1738, 120021 (2016); doi: 10.1063/1.4951904

View online: http://dx.doi.org/10.1063/1.4951904

View Table of Contents: http://aip.scitation.org/toc/apc/1738/1

Published by the American Institute of Physics 


\title{
Converting PSO Dynamics into Complex Network - Initial Study
}

\author{
Michal Pluhacek $^{1}$, Jakub Janostik ${ }^{1}$, Roman Senkerik ${ }^{1}$ and Ivan Zelinka ${ }^{2}$ \\ ${ }^{I}$ Tomas Bata University in Zlín, Faculty of Applied Informatics \\ Department of Informatics and Artificial Intelligence \\ nám. T.G. Masaryka 5555, 76001 Zlín, CZECH REPUBLIC \\ ${ }^{2}$ Department of Computer Science, Faculty of Electrical Engineering and Computer Science \\ VB-TUO, 17. listopadu 15, 70833 Ostrava-Poruba, Czech Republic
}

\begin{abstract}
In this paper it is presented the initial study on the possibility of capturing the inner dynamic of Particle Swarm Optimization algorithm into a complex network structure. Inspired in previous works there are two different approaches for creating the complex network presented in this paper. Visualizations of the networks are presented and commented. The possibilities for future applications of the proposed design are given in detail.
\end{abstract}

Keywords: Particle swarm optimization, Complex network, Inner dynamic, Topology

PACS: 07.05.Tp, 05.45.Pq, 89.20.Ff, 89.75.Fb, 89.75.-k .

\section{INTRODUCTION}

In recent years there has been a significant development in the area of evolutionary computational techniques (ECTs). However the majority of the research is focused on modification of inner principles of the ECTs. Every year many new method and modification are proposed with various successes. In this work it is presented a different approach for enhancing ECTs. The inner dynamic of Particle Swarm Optimization algorithm (PSO) [1 - 4] is captured in the form of complex network [5,6]. Inspired in previous work [6] it is proposed a method to create the network and future applications are outlined.

\section{PARTICLE SWARM OPTIMIZATION ALGORITHM}

The PSO algorithm is inspired in the natural swarm behavior of birds and fish. It was introduced by Eberhart and Kennedy in 1995 [1]. Each particle in the population represents a candidate solution for the optimization problem that is defined by the cost function (CF). In each iteration of the algorithm, a new location (combination of CF parameters) for the particle is calculated based on its previous location and velocity vector (velocity vector contains particle velocity for each dimension of the problem). Within this research the PSO algorithm with global topology (GPSO) [6] was utilized. The chaotic PRNG is used in the main GPSO formula (1), which determines a new "velocity", thus directly affects the position of each particle in the next iteration.

$$
v_{i j}^{t+1}=w \cdot v_{i j}^{t}+c_{1} \cdot \text { Rand } \cdot\left(\text { pBest }_{i j}-x_{i j}^{t}\right)+c_{2} \cdot \text { Rand } \cdot\left(\text { gBest }_{j}-x_{i j}^{t}\right)
$$

Where:

$\mathrm{v}_{i}^{t+1}$ - New velocity of the $i t h$ particle in iteration $t+1$.

$w$ - Inertia weight value; $\mathrm{v}_{i}^{t}$ - Current velocity of the ith particle in iteration $t . ; c_{1}, c_{2}$ - Priority factors; $p B e s t_{\mathrm{i}}-$

Personal best solution found by the $i t h$ particle; $g B e s t$ - Best solution found in a population; $\mathrm{x}_{i j}{ }^{t}$ - Current position of the $i t h$ particle (component $j$ of dimension $D$ ) in iteration $t$; Rand - Pseudo random number, interval $(0,1)$. CPRNG is applied only here.

The maximum velocity was limited to 0.2 times the range as it is usual. The new position of each particle is then given by (2), where $x_{\mathrm{i}}^{\mathrm{t}+1}$ is the new particle position:

$$
x_{i}^{t+1}=x_{i}^{t}+v_{i}^{t+1}
$$

International Conference of Numerical Analysis and Applied Mathematics 2015 (ICNAAM 2015)

AIP Conf. Proc. 1738, 120021-1-120021-4; doi: 10.1063/1.4951904

Published by AIP Publishing. 978-0-7354-1392-4/\$30.00 
Finally the linear decreasing inertia weight $[3,4]$ is used in the typically referred GPSO design that was used in this study. The inertia weight has two control parameters wstart and wend. A new w for each iteration is given by (3), where $\mathrm{t}$ stands for current iteration number and $\mathrm{n}$ stands for the total number of iterations. The values used in this study were wstart $=0.9$ and wend $=0.4$.

$$
w=w_{\text {start }}-\frac{\left(\left(w_{\text {start }}-w_{\text {end }}\right) \cdot t\right)}{n}
$$

\section{EXPERIMENT}

In the initial experiment the PSO algorithm with typical default setting was used to optimize the commonly used Schwefel's benchmark function. The main goal was not the results of the optimization but the recording of inner dynamics of the swarm. Each particle is represented as a node in the network. Furthermore a time-window of 10 iterations was used and therefore there are 10 nodes for each particle (and corresponding time-slot). Using this unique design it is possible to incorporate not only the relations in instant moment but also the influence of time on the dynamic.

For creating the links in the network it is necessary to understand the logic of PSO. The only way of communication that happens in the swarm is the shared knowledge of global best solution. Therefore the particle that found the last gBest is understood to influence all other particles until new gBest is found.

In the first experiment all interactions (improving and non-improving) were tracked. The complex network obtained using this approach is depicted in Fig. 1. The density of such network is very high.

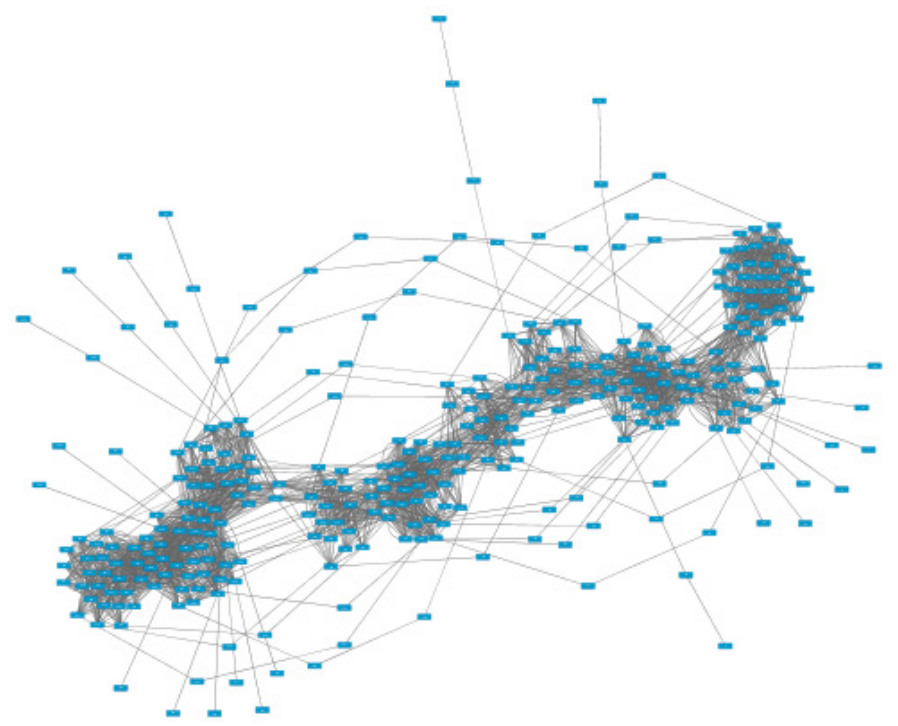

FIGURE 1. PSO dynamic as complex network (10 iterations) - all interactions

In the second experiment only interactions that led to improvement of the particle (in terms of CF) were tracked. The network obtained by this method is completely different and less dense on the first look (See Fig. 2.) but in closer look still contains high amount of information as is clearly demonstrated in Fig. 3. 


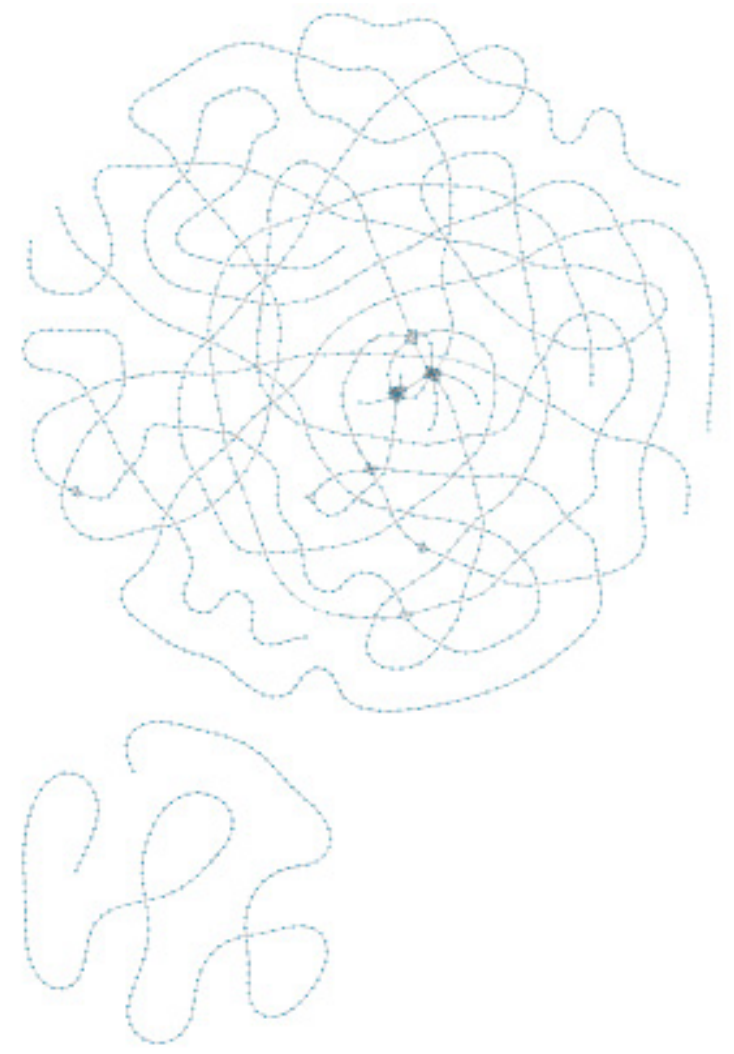

FIGURE 2. PSO dynamic as complex network (10 iterations) - improving interaction only

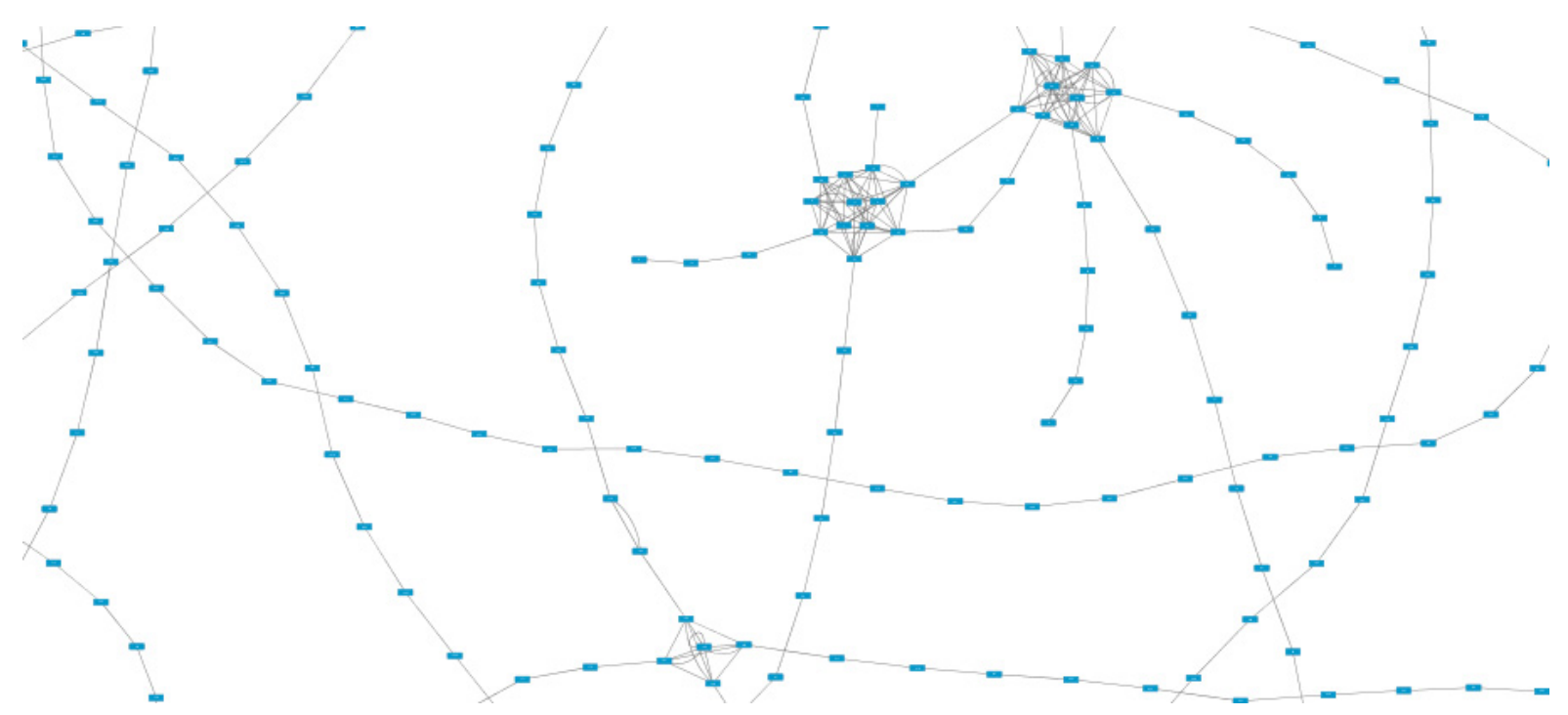

FIGURE 3. PSO dynamic as complex network (10 iterations) - improving interaction only (zoomed) 


\section{FUTURE WORKS}

As has been shown above it is possible to capture the inner dynamic of PSO algorithm in the form of a complex network. Also it has been mentioned that for practical purposes it is necessary to choose a time-window. As application it is understood here that by analyzing the complex network it will be possible to evaluate the performance of the algorithm and the hidden inner dynamics in such way that various adaptive [7] and learning methods can be implemented in order to improve the performance of the algorithm and evade risk of pathological states such as premature convergence or partial/full stagnation.

\section{CONCLUSION}

In this initial study two approaches for capturing the inner dynamic of PSO algorithm into complex network were described. The visualizations of these networks were presented as promising examples. The work will continue with in-deep analysis of the network using both statistical and non-statistical method and approaches. The results of complex network analysis will be used as a vital part of new adaptive approaches. It seems very likely that it is possible to use the complex-network as a tool to understand the otherwise hidden inner dynamics of ECTs.

\section{ACKNOWLEDGMENTS}

This work was supported by Grant Agency of the Czech Republic - GACR P103/15/06700S, further by financial support of research project NPU I No. MSMT-7778/2014 by the Ministry of Education of the Czech Republic. also by the European Regional Development Fund under the Project CEBIA-Tech No. CZ.1.05/2.1.00/03.0089, partially supported by Grant of SGS No. SP2015/142 and SP2015/141, VŠB - Technical University of Ostrava, Czech Republic and by Internal Grant Agency of Tomas Bata University under the project No. IGA/FAI/2015/057 and IGA/FAI/2015/061.

\section{REFERENCES}

1. Kennedy, J., Eberhart, R.: Particle swarm optimization. In: IEEE International Conference on Neural Networks, 1995, pp. 1942-1948.

2. Kennedy, J., Eberhart, R.C., Shi, Y.: Swarm Intelligence. Morgan Kaufmann Publishers, (2001).

3. Nickabadi, A., Ebadzadeh, M.M., Safabakhsh, R.: A novel particle swarm optimization algorithm with adaptive inertia weight. Applied Soft Computing 11(4), 3658-3670 (2011).

4. Yuhui, S., Eberhart, R.: A modified particle swarm optimizer. In: IEEE World Congress on Computational Intelligence., 4-9 May 1998, pp. 69-73.

5. Boccaletti S., Latora V., Moreno Y., Chavez M., and Hwang D.-U., “Complex Networks: Structure and Dynamics,”Physics Reports,424(4-5), 2006 pp. 175-308. doi:10.1016/j.physrep.2005.10.009.

6. Zelinka, I., Davendra, D., Enkek, R., Jaek, R.: Do Evolutionary Algorithm Dynamics Create Complex Network Structures? Complex Systems 2, 0891-2513, 20, 127-140

7. J Riget, J S Vestterstrom. A Diversity-guided particle swarm optimizer ${ }^{\vee}$ the ARPSO. Technical report, EVAlife, Dept. of Computer Science, University of Aarhus, Denmark, 2002 